
\title{
28 Research Soure \\ Temperature Environmental Adaptability Research of HNIW/FOX-7 Based PBXs
}

\section{Guanchao Lan ( $\nabla$ gclan@nuc.edu.cn )}

North University of China

\section{Hui Chao}

Gansu Yin Guang Chemical Industry Group Co. Ltd.

\section{Na Wang}

Gansu Yin Guang Chemical Industry Group Co. Ltd.

\section{Bo Yan}

Gansu Yin Guang Chemical Industry Group Co. Ltd.

\section{Xiao Li}

North University of China

Shaohua Jin

Beijing Institute of Technology

\section{Research Article}

Keywords: temperature adaptability, HNIW/FOX-7 based PBXs, mechanical properties, thermal decomposition

Posted Date: September 23rd, 2021

DOl: https://doi.org/10.21203/rs.3.rs-900463/v1

License: (c) (1) This work is licensed under a Creative Commons Attribution 4.0 International License.

Read Full License 


\section{Abstract}

In this study, HNIW/FOX-7 based PBX modeling powders and PBX columns were treated by LT (low temperature), HT (high temperature), HLC (high-low temperature cycle) and HLS (high-low temperature shock) to study temperature environmental adaptability of HNIW/FOX-7 based PBXs. Then SEM, IR, XRD and DSC were used to study the variation of PBX modeling powders after $L T, H T, H L C$ and HLS treatments; in addition, the mass, size and mechanical properties of PBX columns were characterized after different temperature adaptability treatments as well. The results indicate that the change ratios of mass and size of HNIW/FOX-7 based PBX columns are less than 1\%, illustrating that mass and size of PBX columns are at acceptable level after different temperature adaptability treatments. The unevenness degree of the surface of PBX modeling powders followed the order of HLC>HT>LT>HLS, which agrees well with mass loss order. Moreover, IR and XRD results indicated that the molecular structure and crystal form of HNIW and FOX-7 did not change after different temperature adaptability treatments. Additionally, thermal stabilities of PBX modeling powders are decreased after different temperature adaptability treatments, among which HLS has the largest influence on HNIW/FOX-based PBX modeling powders. The compression strengths and elastic moduli of HNIW/FOX-based PBX columns are enhanced after different temperature adaptability treatments, among which the strength of PBX columns after HLC has the maximum increase, indicating that HLC has more significant effect on mechanical property.

\section{Introduction}

2,4,6,8,10,12-Hexanitro-2,4,6,8,10,12-hexaazaisowurtzitane (HNIW) with cage structure has been widely used as the main explosive in many high energy polymer bonded explosives (PBXs) formulations [1-3] because of its high energy density, high detonation pressure and high detonation velocity [4,5]. However, energy and security are often conflicting to each other. High energy of HNIW based PBXs bring high sensitivity and hazards as well. Nowadays, the sensitivity and vulnerability of high energy explosives are expected to be reduced for enhancing the security of modern weaponry. In order to achieve this goal, it's a good technique by using insensitive explosive such as 1,1-diamino-2,2-dinitroethylene (FOX-7) to replace some HNIW to prepare HNIW/FOX-7 based high energy low vulnerability PBXs. By adjusting the content of FOX-7, the energy and security of HNIW/FOX-7 based PBXs can be balanced $[6,7]$.

Explosives will undergo various temperature environments during their storage, transportation and application. The complex temperature environments may change the mass, size and detonation properties of munitions, which may produce damage and aging inside munitions and exert effects on energy, security and mechanical properties of PBXs, and then produce negative influence on overall functionality of weapon system [8]. In addition, HNIW have more than one kind of crystalline form, and different crystalline form can be converted to each other at high temperature, and FOX-7 has the same phenomenon. The various temperature environments may change the crystalline form of HNIW and FOX7, which may result in great variation of properties of HNIW/FOX-7 based PBXs. Therefore, the investigation of temperature adaptability of HNIW/FOX-7 based PBXs is imperative. 
In this study, aqueous suspension method was adopted to prepare HNIW/FOX-7 based PBX modeling powders. Then, HNIW/FOX-7 based PBX modeling powders and PBX columns were treated by LT (low temperature), HT (high temperature), HLC (high-low temperature cycle) and HLS (high-low temperature shock) to study temperature environmental adaptability of HNIW/FOX-7 based PBXs. After LT, HT, HLC and HLS treatments, SEM, IR, XRD and DSC were used to study the variation of PBX modeling powders. In addition, the mass, size and mechanical properties of PBX columns were characterized after HT, LT, HLC and HLS treatments as well. By comparing the variation SEM, IR, XRD, DSC, mass, size and mechanical properties after different temperature adaptability treatments, the temperature adaptability of HNIW/FOX7 based PBXs are obtained.

\section{Experimental Details}

\subsection{Preparation of HNIW/FOX-7 based PBX samples}

Water suspension method $[9,10]$ was adopted to prepare HNIW/FOX-7 based PBX modeling powders (47 wt\% HNIW, 47 wt\% FOX-7, 5 wt\% binder system, 0.5 wt\% wax, 0.5 wt $\%$ graphite). The PBX modeling powders prepared in this study are displayed in Fig. 1a. Then, the modeling powders were further pressed to $\Phi 20 \times 20 \mathrm{~mm}$ PBX columns using hydraulic press with the temperature and loading rate of $25^{\circ} \mathrm{C}$ and 1 $\mathrm{kN} \cdot \mathrm{s}^{-1}$ respectively. When the pressure of hydraulic press reached the maximum values $\left(2000 \mathrm{~kg} \cdot \mathrm{cm}^{-2}\right)$, maintain the maximum pressure for $10 \mathrm{~min}$. Adopting these conditions, the real density of HNIW/FOX-7 based PBX columns could reach $97 \%$ of the theoretical maximum density. The PBX columns prepared in this study are displayed in Fig. $1 \mathrm{~b}$.

\subsection{Temperature adaptability treatments}

In this study, using constant humidity-temperature chamber (QS-GS100FB), HNIW/FOX-7 based PBXs are treated under $L T, H T, H L S$ and $H L C$, respectively $[11,12]$. Temperature variation settings of $L T, H T, H L S$ and HLC are shown in Fig. 2.

At ambient temperature, 10 grams PBX modeling powders and 5 PBX columns were placed into constant humidity-temperature chamber. The temperature of chamber was froze to $-55^{\circ} \mathrm{C}$ with a cooling rate of 2 ${ }^{\circ} \mathrm{C} \cdot \mathrm{min}^{-1}$ and maintained for 24 hours to study the effect of LT on the performance of HNIW/FOX-7 based PBX. The temperature of chamber was heated to $71{ }^{\circ} \mathrm{C}$ with a heating rate of $2{ }^{\circ} \mathrm{C} \cdot \mathrm{min}^{-1}$ and maintained for 48 hours to study the effect of HT on the performance of HNIW/FOX-7 based PBX. The temperature of chamber was heated to $71{ }^{\circ} \mathrm{C}$ and maintained for 1 hour, and then the temperature was froze to $-55^{\circ} \mathrm{C}$ and maintained for 1 hour. During the test, the heating and cooling rate was controlled at $20{ }^{\circ} \mathrm{C} \cdot \mathrm{min}^{-1}$. Repeat this cycle three time to study the effect of HLS on the performance of HNIW/FOX-7 based PBX. HNIW/FOX-7 based PBX samples were placed in temperature cycle between $-55^{\circ} \mathrm{C}$ and $71^{\circ} \mathrm{C}$ for five cycles to study the effect of HLC on PBX performance. For each cycle, the room temperature $\left(30^{\circ} \mathrm{C}\right)$, low temperature $\left(-55^{\circ} \mathrm{C}\right)$ and high temperature $\left(71^{\circ} \mathrm{C}\right)$ were maintained for 2,313 hours respectively. The heating and cooling rate was controlled at $0.67^{\circ} \mathrm{C} \cdot \mathrm{min}^{-1}$ during temperature variation process. 


\subsection{Characterizations}

\subsubsection{Mass and size of PBX columns}

After LT, HT, HLS and HLC treatments, the mass and size (diameter and height) of the PBX columns were measured using analytical balance and screw micrometer, respectively. The average variation ratio of mass and size of 5 PBX columns were obtained.

\subsubsection{Surface micro-topography}

After LT, HT, HLS and HLC treatments, the surface micro-topography of PBX modeling powders was obtained by scanning electron microscope (SEM, MIRA3 XM, Tescan Co., Ltd., Brno, Czech Republic).

\subsubsection{Infrared spectrum (IR)}

After LT, HT, HLS and HLC treatments, IR spectra of PBX modeling powders were recorded using $\mathrm{KBr}$ plates on a Nicolet 6700 spectrometer produced by Thermo Fisher Scientific. The wavenumber ranges from 4000 to $400 \mathrm{~cm}^{-1}$, and the tests were carried out at room temperature.

\subsubsection{X-ray diffraction (XRD)}

After LT, HT, HLS and HLC treatments, X-ray powder diffraction of PBX modeling powders were collected on a Rigaku Ultima $₫$ which was made by Beijing Glory Leader Technology Co., Ltd. The test voltage and electric current were $40 \mathrm{kV}$ and $44 \mathrm{~mA}$, respectively. Scanning angle ranges from 10 to $70^{\circ}$ with the scanning speed of $2 \%$ min.

\subsubsection{Thermal decomposition}

After $L T, H T, H L S$ and HLC treatments, thermal decomposition behavior of PBX modeling powders was measured using differential scanning calorimeter (DSC 200 F3, aluminum crucible with a pin hole cover). The sample with the mass of $0.70 \pm 0.01 \mathrm{mg}$ was heated from $50^{\circ} \mathrm{C}$ to $320^{\circ} \mathrm{C}$ under nitrogen with a flow rate of $70 \mathrm{~mL} \cdot \mathrm{min}^{-1}$ with a heating rate of $0.5,1,1.5$ and $2^{\circ} \mathrm{C} \cdot \mathrm{min}^{-1}$, respectively. Each sample was analyzed three times.

To obtain the kinetics parameters of the main exothermic decomposition reactions, Kissinger [13] and Ozawa [14] methods, expressed in Eqs. (1) and (2), respectively, were adopted.

$\ln \left(\frac{\beta}{T_{\mathrm{P}}^{2}}\right)=\ln \left(\frac{A R}{E_{a}}\right)-\frac{E_{a}}{R T_{\mathrm{P}}}$

$\lg \beta=\lg \left[\frac{A E_{a}}{R G(\alpha)}-2.315-0.4567 \frac{E_{a}}{R T_{\mathrm{P}}}\right]$

where $\beta$ is the heating rate $\left({ }^{\circ} \mathrm{C} \cdot \mathrm{min}^{-1}\right), T_{\mathrm{p}}$ is the peak temperature $(\mathrm{K}), E_{\mathrm{a}}$ is the apparent activation energy $\left(\mathrm{kJ} \cdot \mathrm{mol}^{-1}\right), A$ is the pre-exponential factor $\left(\mathrm{s}^{-1}\right), R$ is the gas constant valued as $8.314 \mathrm{~J} \cdot \mathrm{mol}^{-1} \cdot \mathrm{K}^{-1}, a$ is 
the conversion degree that was the mass ratio of the reacted substance to the raw, and $G(a)$ is the integral mechanism function.

\subsubsection{Adiabatic accelerating thermal decomposition}

After LT, HT, HLS and HLC treatments, adiabatic thermal decomposition behavior of PBX modeling powders was studied using accelerating rate calorimeter (ARC, NETZSCH 254) instrument. $110 \pm 1 \mathrm{mg}$ samples, spherical hastelloy $\mathrm{C}$ vessel, and 'heat-wait-search' procedure were used during the measurement. The temperature range of ARC experiment is between $50{ }^{\circ} \mathrm{C}$ and $230{ }^{\circ} \mathrm{C}$. Based on the measured results, the kinetics parameters and mechanism function of PBX modeling powders adiabatic thermal decomposition are obtained.

\subsubsection{Mechanical property}

After LT, HT, HLS and HLC treatments, according to GJB 772A-97 [15], the compression strength $\left(\sigma_{\mathrm{c}}\right)$ and elastic modulus $(E)$ of PBX columns are analyzed using electromechanical universal testing machine (CMT4502). Each PBX column is imposed a quasi-static compression load along the axial with a loading rate of $0.5 \mathrm{~mm} \cdot \mathrm{min}^{-1}$ until electromechanical universal testing machine reach the maximum load (4.5 $\mathrm{kN}$ ) or PBX column was damaged. Then $E$ and $\sigma_{\mathrm{c}}$ could be calculated by the following two equations,

$\varepsilon=\frac{\sigma}{E}+A \sigma^{m}$

$\sigma_{\mathrm{c}}=\frac{4 P}{\pi d^{2}}$

where $\varepsilon$ and $\sigma$ are strain and stress respectively, $A$ and $m$ are model parameters, $P$ is the compression load and $d$ is the diameter of PBX column.

\section{Results And Discussion}

\subsection{Mass and size}

Table 1 lists the change ratios of mass and size (height and diameter) of HNIW/FOX-7 based PBX columns. The results illustrate that the change ratios of mass and size (height and diameter) of PBX columns are within 1\%. Based on American military standard MIL-STD-1751 [16], the mass and size of PBX columns are still at acceptable level after different temperature adaptability treatments.

After LT, HT, HLS and HLC treatments, the mass of each PBX column is declined, and the variation ratio of the mass of PBX columns after different temperature adaptability treatments follow the order of HLC > $\mathrm{HT}>\mathrm{LT}>\mathrm{HLS}$, which is associated with the treatment time of temperature adaptability treatments. The variation of the size of PBX columns is more complex than that of mass. It can be concluded that all the diameters are decreased, but the heights are not totally increased or decreased. On the one hand, the mass loss of PBX columns may cause the variation of size. On the other hand, the variation of 
temperature affects the motion ability of PBX molecules may cause the variation of size as well. The densities of PBX columns are increased after different temperature adaptability treatments, in that the internal stress of PBX columns are released resulting in the decrease of volume. The change ratios of PBX columns after different temperature adaptability treatments are far less than the failure criterion. Therefore, the influence of LT, HT, HLS and HLC on the mass and size of HNIW/FOX-7 based PBX columns can be neglected.

Table 1

Variation ratios of mass and size of the HNIW/FOX-7 based PBX columns.

\begin{tabular}{|l|lllll|}
\hline Test & $(\Delta d / d) / \%$ & $(\Delta h / h) / \%$ & $(\Delta \rho / \rho) / \%$ & $(\Delta m / m) / \%$ \\
\hline HT & -0.099 & 0.078 & 0.213 & -0.093 \\
LT & -0.050 & 0.000 & 0.152 & -0.052 \\
HLC & -0.129 & 0.185 & 0.182 & -0.108 \\
HLS & -0.139 & -0.039 & 0.356 & -0.040 \\
\hline
\end{tabular}

SEM is used to study the surface micro-topography of HNIW/FOX-based PBX modeling powders. Figure 3 displays the surface micro-topography of PBX modeling powders before and after LT, HT, HLS and HLC treatments. Figure 3 illustrates that the coating effect of untreated samples is excellent for almost no explosives crystal are exposed. However, after different temperature adaptability treatments, the surface of PBX modeling powders become uneven and emerge many cavities, which is probably caused by the sublimation and gasification of the additives. In addition, it is can be concluded from Fig. 3 that the unevenness degree of the surface of PBX modeling powders follows the order of HLC $>H T>L T>H L S$, which agrees well with variation ratios of mass loss.

\subsection{Molecular structure}

To investigate whether the structures of HNIW/FOX-based PBX samples have changed after LT, HT, HLS and HLC treatments, the samples are characterized by IR spectroscopy (Fig. 4). It can be concluded from Fig. 4 that after different temperature adaptability treatments all IR spectroscopies are consistent with the untreated sample, indicating that the structures of HNIW/FOX-based PBX samples have not changed.

\subsection{Crystalline form}

HNIW and FOX-7 exist polymorphs [17-19], and their crystalline form may transform at high temperature. To research whether the crystalline form of HNIW and FOX-7 in HNIW/FOX-7 based PBX has changed after LT, HT, HLS and HLC treatments, the samples are characterized by XRD (Fig. 5). Figure 5 shows that after different temperature adaptability treatments all XRD spectroscopies are consistent with the untreated sample, indicating that the crystal form of HNIW and FOX-7 in HNIW/FOX-7 based PBX samples has not changed. 


\subsection{Mechanical properties}

After different temperature adaptability treatments, the compression strengths and elastic moduli of PBX columns are investigated. The stress-strain curves of PBX columns are displayed in Fig. 6 , and the calculated compression strengths and elastic moduli are listed in Table 2.

Table 2

Compression strengths and elastic moduli of the HNIW/FOX-7 based

PBX columns.

\begin{tabular}{|lll|}
\hline Treatments & $\sigma_{\mathrm{c}} / \mathrm{MPa}$ & E/MPa \\
\hline Untreated & 8.07 & 2.30 \\
\hline HT & 10.96 & 3.01 \\
\hline LT & 8.38 & 2.47 \\
\hline HLC & 11.14 & 3.17 \\
\hline HLS & 10.11 & 2.78 \\
\hline
\end{tabular}

It can be concluded from Table 2 that the compression strengths and elastic moduli of the PBX columns are all increased after HT, LT, HLC and HLS, indicating the enhance of rigidity of PBX columns. The variation of $\sigma_{\mathrm{c}}$ and $E$ after $\mathrm{LT}$ is relatively small, demonstrating that the effects of low temperature on HNIW/FOX-7 based PBX columns are small. However, the influence of HLC on HNIW/FOX-7 based PBX columns are significant. Although the content of binder system is low in this HNIW/FOX-7 based PBX formulation, it may repair some defects induced by pressing because of its fluidity and spreadability during storage, thus increasing the mechanical properties of the PBX columns. In addition, oxidative crosslinking reaction of binder may occur after temperature adaptability tests under the influence of temperature, which increases the crosslinking point of the polymer network formed after reaction and then raise the crosslinking density, and finally improves compression properties and elastic moduli.

\subsection{Thermal decomposition}

After different temperature adaptability treatments, thermal decomposition performance of the HNIW/FOX-7 based PBX samples are studied by DSC. The measured DSC curves are displayed in Fig. 7. The initial decomposition temperature $\left(T_{0}\right)$, peak temperatures $\left(T_{p}\right)$ and released heat $(\Delta H)$ obtained by DSC measurements are summarized in Table 3. 
Table 3

The initial decomposition temperature (To), peak temperatures

$(\mathrm{Tp})$ and released heat $(\Delta \mathrm{H})$ of PBX samples.

\begin{tabular}{|c|c|c|c|c|}
\hline Treatments & $\beta /{ }^{\circ} \mathrm{C} \cdot \min ^{-1}$ & $T_{0} /{ }^{\circ} \mathrm{C}$ & $T_{\mathrm{p}} /{ }^{\circ} \mathrm{C}$ & $\Delta H / \mathrm{J} \cdot \mathrm{g}^{-1}$ \\
\hline \multirow[t]{4}{*}{ Untreated } & 0.5 & 204.54 & 217.07 & -1608 \\
\hline & 1.0 & 209.11 & 224.16 & -1647 \\
\hline & 1.5 & 212.09 & 227.11 & -1696 \\
\hline & 2.0 & 213.93 & 230.27 & -1778 \\
\hline \multirow[t]{4}{*}{ HLC } & 0.5 & 204.33 & 217.07 & -1590 \\
\hline & 1.0 & 207.82 & 224.58 & -1612 \\
\hline & 1.5 & 210.06 & 228.29 & -1677 \\
\hline & 2.0 & 212.19 & 230.98 & -1744 \\
\hline \multirow[t]{4}{*}{ HLS } & 0.5 & 203.79 & 217.60 & -1583 \\
\hline & 1.0 & 206.89 & 223.76 & -1626 \\
\hline & 1.5 & 210.62 & 228.05 & -1690 \\
\hline & 2.0 & 212.22 & 231.55 & -1748 \\
\hline \multirow[t]{4}{*}{ LT } & 0.5 & 203.59 & 217.23 & -1587 \\
\hline & 1.0 & 206.97 & 224.23 & -1634 \\
\hline & 1.5 & 209.65 & 228.78 & -1686 \\
\hline & 2.0 & 212.55 & 231.38 & -1756 \\
\hline \multirow[t]{4}{*}{ HT } & 0.5 & 203.81 & 217.19 & -1551 \\
\hline & 1.0 & 207.04 & 223.92 & -1624 \\
\hline & 1.5 & 210.59 & 228.29 & -1676 \\
\hline & 2.0 & 212.22 & 230.69 & -1759 \\
\hline
\end{tabular}

Table 3 shows that at the same heating rate $T_{0}$ and $\Delta H$ of PBX samples are decreased after HT, LT, HLC and HLS treatments, illustrating that the thermal insulation effect of binder system and desensitizer on PBX samples may be weakened, resulting in a decrease of PBX samples thermal stability. The decreasing $\Delta H$ reveals that the detonation heat may decline after temperature adaptability treatment. The variation of $T_{\mathrm{p}}$ of PBX samples is more complicated, but the variation ratios are lower than $2{ }^{\circ} \mathrm{C}$ illustrating that the thermal stabilities of HNIW/FOX-7 based PBXs unchanged after diifernt temperature adaptability treatments. 
The kinetics parameters $\left(E_{\mathrm{a}}\right.$ and $A$ ) of HNIW/FOX-7 based PBX samples are calculated using Kissinger and Ozawa methods. The calculated $E_{\mathrm{a}}, A$ and correlation coefficient $\left(R^{2}\right)$ are listed in Table 4 . We can find that the calculated $E_{\mathrm{a}}$ using two different methods are close to each other and the $R^{2}$ are higher than 0.99 , illustrating the calculated kinetics parameters are accurate. Generally, materials with higher value of $E_{\mathrm{a}}$ will be more stable. Table 4 shows that the $E_{\mathrm{a}}$ declined after different temperature adaptability treatments, which indicates a probable decline of the stability of HNIW/FOX-7 based PBXs.

Table 4

The kinetic parameters of HNIW/FOX-7 based PBX samples.

\begin{tabular}{|llllll|}
\hline Sample & \multicolumn{1}{l}{ Kissinger } & \multicolumn{3}{c|}{ Ozawa } \\
\cline { 2 - 6 } & $E_{\mathrm{a}} / \mathrm{kJ}^{\prime} \mathrm{mol}^{-1}$ & $\boldsymbol{A} / \mathbf{s}^{-1}$ & $\boldsymbol{R}^{2}$ & $E_{\mathrm{a}} / \mathrm{kJ} \cdot \mathrm{mol}^{-1}$ & $\boldsymbol{R}^{2}$ \\
\hline Untreated & 209.803 & $1.074 \times 10^{19}$ & 0.9952 & 207.368 & 0.9956 \\
\hline HLC & 195.753 & $3.76 \times 10^{17}$ & 0.9972 & 194.879 & 0.9974 \\
\hline HLS & 197.283 & $9.77 \times 10^{17}$ & 0.996 & 196.381 & 0.9963 \\
\hline HT & 199.827 & $1.25 \times 10^{17}$ & 0.9987 & 198.855 & 0.9988 \\
\hline LT & 190.964 & $1.20 \times 10^{17}$ & 0.9991 & 190.209 & 0.9991 \\
\hline
\end{tabular}

\subsection{Adiabatic accelerating thermal decomposition}

The adiabatic decomposition properties of HNIW/FOX-7 based PBX samples are studied by ARC instrument after different temperature adaptability treatments. The measured initial decomposition temperature $\left(T_{\mathrm{o}}\right)$, final decomposition temperature $\left(T_{\mathrm{f}}\right)$, adiabatic temperature rise $\left(\Delta T_{\mathrm{ad}}\right)$, maximum pressure $\left(P_{\mathrm{m}}\right)$ and released heat $(Q)$ are listed in Table 5. The measured ARC curves are displayed in Fig. 8 , and the variation of temperature $(T)$, pressure $(P)$, temperature change rate $(\mathrm{d} T / \mathrm{d} t)$ and pressure change rate $(\mathrm{d} P / \mathrm{d} t)$ with time $(t)$ during the adiabatic decomposition process of untreated samples are depicted in Fig. 8 as well. 
Table 5

ARC measured adiabatic thermal decomposition parameters.

\begin{tabular}{|llllll|}
\hline Parameters & Untreated & LT & HT & HLC & HLS \\
\hline$T_{0} /{ }^{\circ} \mathrm{C}$ & 181.66 & 181.28 & 181.32 & 181.59 & 180.54 \\
\hline$T_{\mathrm{f}} /{ }^{\circ} \mathrm{C}$ & 222.14 & 218.81 & 219.94 & 220.46 & 219.43 \\
\hline$\Delta T_{\mathrm{ad}} /{ }^{\circ} \mathrm{C}$ & 40.48 & 37.53 & 38.62 & 38.87 & 38.89 \\
\hline$P_{\mathrm{m}} / \mathrm{kPa}$ & 1422.36 & 1354.27 & 1310.10 & 1248.18 & 1394.01 \\
\hline $\mathrm{Q} / \mathrm{J} \cdot \mathrm{g}^{-1}$ & 37.70 & 34.95 & 35.97 & 36.20 & 36.22 \\
\hline
\end{tabular}

ARC measured results illustrate that the initial decomposition temperature of each sample is around 180 ${ }^{\circ} \mathrm{C}$ demonstrating that temperature adaptability treatments have few influences on the adiabatic decomposition stability of HNIW/FOX-7 based PBXs. In addition, the $\Delta T_{\mathrm{ad}}, P_{\mathrm{m}}$ and $Q$ are decrease after HLC, HLS, HT and LT treatments, but the decrease ratios are low, illustrating that temperature adaptability treatments have few influences on the adiabatic decomposition stability of PBXs as well.

According to ARC measured results, the activation energies $\left(E_{\mathrm{a}}\right)$, pre-exponential factors $(A)$ and mechanism functions $(f(a))$ of HNIW/FOX-7 based PBXs adiabatic decomposition are calculated using mechanism functions method [20-22]. The calculated results are summarized in Table 6.

Table 6

The calculated $E_{a}, A$ and $f(a)$ of adiabatic decomposition.

\begin{tabular}{|lllll|}
\hline samples & $E_{\mathrm{a}} / \mathrm{kJ} \cdot \mathrm{mol}^{-1}$ & $A / \mathbf{s}^{-1}$ & $f(a)$ & $R^{2}$ \\
\hline Untreated & 290.458 & $3.594 \times 10^{30}$ & $(1-a)^{2}$ & 0.992 \\
HLC & 273.162 & $8.767 \times 10^{26}$ & $(1-a)^{2}$ & 0.991 \\
HLS & 281.679 & $1.063 \times 10^{27}$ & $(1-a)^{2}$ & 0.984 \\
HT & 278.851 & $8.902 \times 10^{26}$ & $(1-a)^{2}$ & 0.986 \\
LT & 284.713 & $9.134 \times 10^{27}$ & $(1-a)^{2}$ & 0.993 \\
\hline
\end{tabular}

It can be deduced from Table 6 that $f(a)$ are still $(1-a)^{2}$ after different temperature adaptability treatments, illustrating HLC, HLS, HT and LT treatments wouldn't change the mechanism functions of HNIW/FOX-7 based PBXs. $E_{\mathrm{a}}$ decreased $17.30 \mathrm{~kJ} \cdot \mathrm{mol}^{-1}(5.95 \%), 8.78 \mathrm{~kJ} \cdot \mathrm{mol}^{-1}(3.02 \%), 11.61 \mathrm{~kJ} \cdot \mathrm{mol}^{-1}(4.00 \%)$ and 5.75 $\mathrm{kJ} \cdot \mathrm{mol}^{-1}(1.98 \%)$, respectively after HLC, HLS, HT and LT treatments. It can be also concluded that the variation ratios of $E_{\mathrm{a}}$ is related to the treatments time of high temperature. After HLC, HLS, HT and $\mathrm{LT}$ treatments, the variation ratios of $E_{\mathrm{a}}$ are lower than $20 \%$, which are still in an acceptable level according to GJB 772A-97. 


\section{Conclusions}

In this study, HNIW/FOX-7 based PBX samples were treated by LT, HT, HLC and HLS. Then the mass, size, surface micro-topography, molecular structure, crystalline form, mechanical property and thermal decomposition are characterized to study temperature environmental adaptability of HNIW/FOX-7 based PBXs. The main conclusions can be summarized as follows:

(1) The change ratios of mass and size (diameter and height) of HNIW/FOX-7 based PBX columns are lower than $1 \%$, indicating that PBX columns are still at an acceptable level after different temperature adaptability treatments.

(2) The surface micro-topography of PBX samples become uneven and emerge many cavities, which is probably caused by the sublimation and gasification of the additives. The unevenness degree of the surface of PBX modeling powders follows the order of HLC $>H T>L T>H L S$, which agrees well with variation ratios of mass loss.

(3) The characterization results of IR and XRD demonstrate that the structure and crystal form of HNIW/FOX-7 based PBXs have not changed after temperature adaptability treatments.

(4) The compression strengths and elastic moduli of PBX columns are increased after HT, LT, HLC and HLS treatments, indicating the enhance of rigidity of PBX columns.

(5) HT, LT, HLC and HLS treatments have few influences on the initial decomposition temperatures, activation energies, pre-exponential factors $(A)$ and mechanism functions of HNIW/FOX-7 based PBXs, indicating that the thermal stabilities of PBXs are almost unchanged.

Above all, after HT, LT, HLC and HLS treatments the performances of HNIW/FOX-7 based PBXs have no significant change, illustrating the temperature environmental adaptabilities of HNIW/FOX-7 based PBXs are adaptable.

\section{References}

1. Li, J. \& Brill, T. B. Nanostructured energetic composites of CL-20 and binders synthesized by sol gel methods. Propell Explos Pyrot, 31, 61-69 (2006).

2. Thiboutot, S., Brousseau, P., Ampleman, G., Pantea, D. \& Côté, S. Potential use of CL-20 in TNT/ETPEbased melt cast formulations. Propell Explos Pyrot, 33, 103-108 (2008).

3. Samudre, S. S. et al. Studies on an improved plastic bonded explosive (PBX) for shaped charges. Propell Explos Pyrot, 34, 145-150 (2009).

4. Ou, Y. X. Explosives (Beijing Institute of Technology Press, Beijing, 2014).

5. Lan, G. C. et al. The study of external growth environments on the crystal morphology of $\varepsilon-H N I W$ by molecular dynamics simulation. J Mater Sci, 53, 12921-12936 (2018). 
6. Joseph, M. D. et al. Studies on Advanced RDX/TATB based low vulnerable sheet explosives with HTPB binder. Propell Explos Pyrot, 34, 326-330 (2009).

7. Becuwe, A. \& Deltlos, A. Low-sensitivity explosive compounds for low vulnerability warheads. Propell Explos Pyrot, 18, 1-10 (1993).

8. Yin, J. T., Yuan, B. H., Shi, W. W., Wang, J. C. \& Gao, Y. L. Aging damage and mechanical environment adaptability of pressed HMX-based PBX. Chin J Energ Mater, 24, 674-677 (2016).

9. Elbeih, A., Pachman, J., Zeman, S., Trzcinski, W. A. \& Suceska, M. Study of plastic explosives based on attractive cyclic nitramines: part II. Detonation characteristics of explosives with polyfluorinated binders. Propell Explos Pyrot, 38, 238-243 (2013).

10. Niu, H., Chen, S. S., Shu, Q. H., Li, L. J. \& Jin, S. H. Preparation, characterization and thermal risk evaluation of dihydroxylammonium 5, 5-bistetrazole-1, 1-diolate based polymer bonded explosive. $J$ Hazard Mater, 338, 208-217 (2017).

11. Lan, G. C. et al. Investigation into the temperature adaptability of HNIW-based PBXs. Propell Explos Pyrot, 44, 327-336 (2019).

12. Wei, X. W., Zhou, X. Y., Xu, X. Z. \& Wang, P. Thermal environment adaptability of HMX based PBX. Chin J Explos Propell, 35, 15-17 (2012).

13. Kissinger, H. E. Reaction kinetics in different thermal analysis. Anal Chem, 29, 1702-1706 (1957).

14. Ozawa, T. A new method of analyzing thermogravimetric data. B Chem Soc Jpn, 38, 1881-1886 (1965).

15. GJB 772A-97 (1997) Explosive Test Method, Beijing, China.

16. MIL-STD-1751 (1982) Military standard: safety and performance tests for qualification of explosives.

17. Nielsen, A. T. et al. Synthesis of polyazapolycyclic caged polynitramines., 54, 11793-11812 (1998).

18. Russell, T. R., Miller, P. J., Piermarini, G. J. \& Block, S. High-pressure phase transition in gammahexanitrohexaazaisowurtzitane. J Phys Chem, 96, 5509-5512 (1992).

19. Foltz, M. F. Thermal stability of $\varepsilon$-hexanitrohexaazaisowurtzitane in an estane formulation. Propell Explos Pyrot, 19, 63-69 (1994).

20. Lan, G. C. et al. Preparation and performances characterization of HNIW/NTO-based high-energetic low vulnerable polymer-bonded explosive. J Therm Anal Calori, 139, 3589-3602 (2020).

21. Zhang, G. Y. et al. Evaluation of thermal hazards and thermo-kinetic parameters of 3-amino-4amidoximinofurazan by ARC and TG. J Therm Anal Calorim, 126, 1223-1230 (2016).

22. Townsend, D. I. \& Tou, J. C. Thermal hazard evaluation by an accelerating rate calorimeter. Thermochim Acta, 37, 1-30 (1980).

\section{Figures}




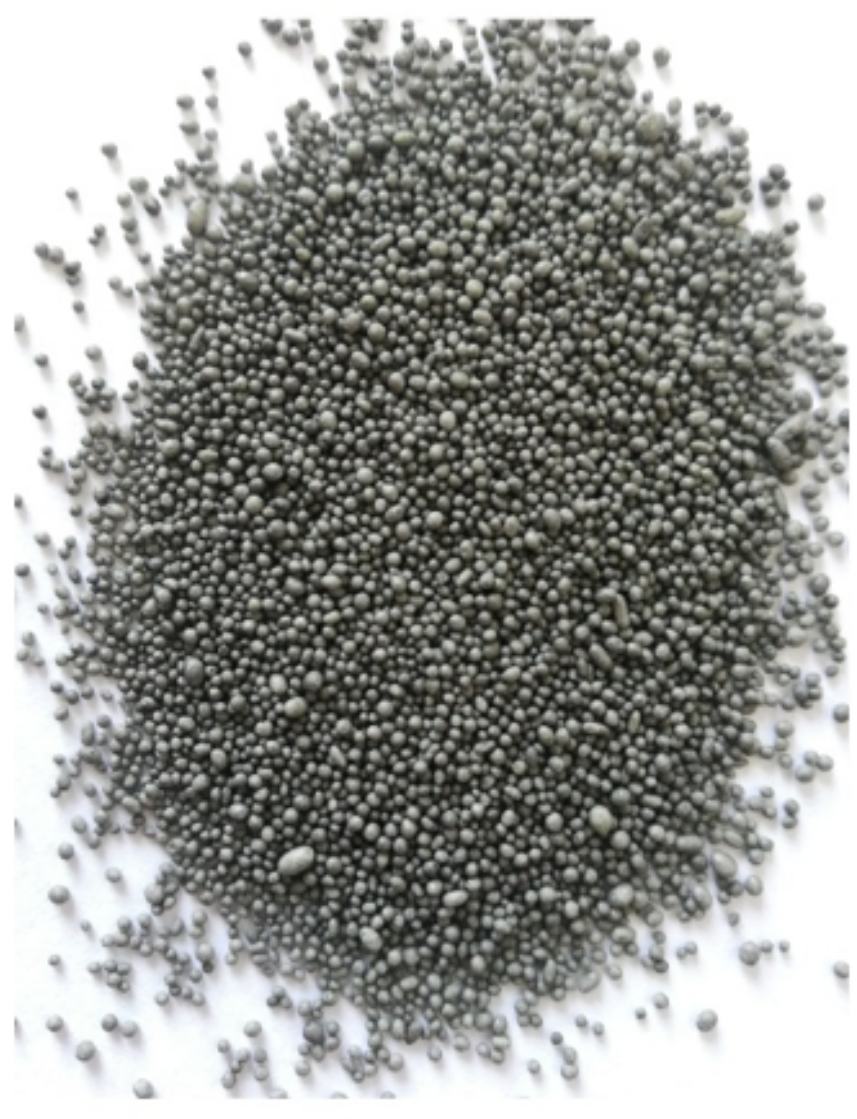

a

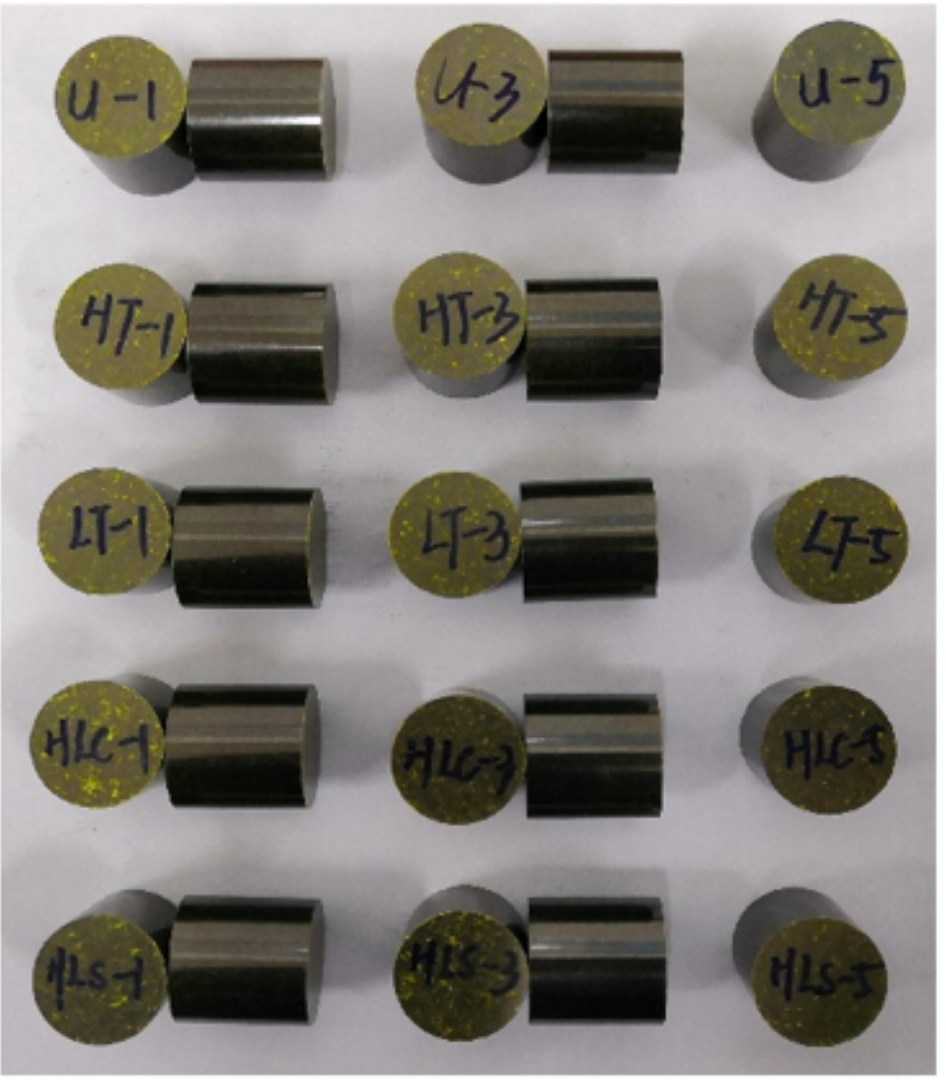

b

\section{Figure 1}

a HNIW/FOX-7 based PBX modeling powders, b HNIW/FOX-7 based PBX columns. 


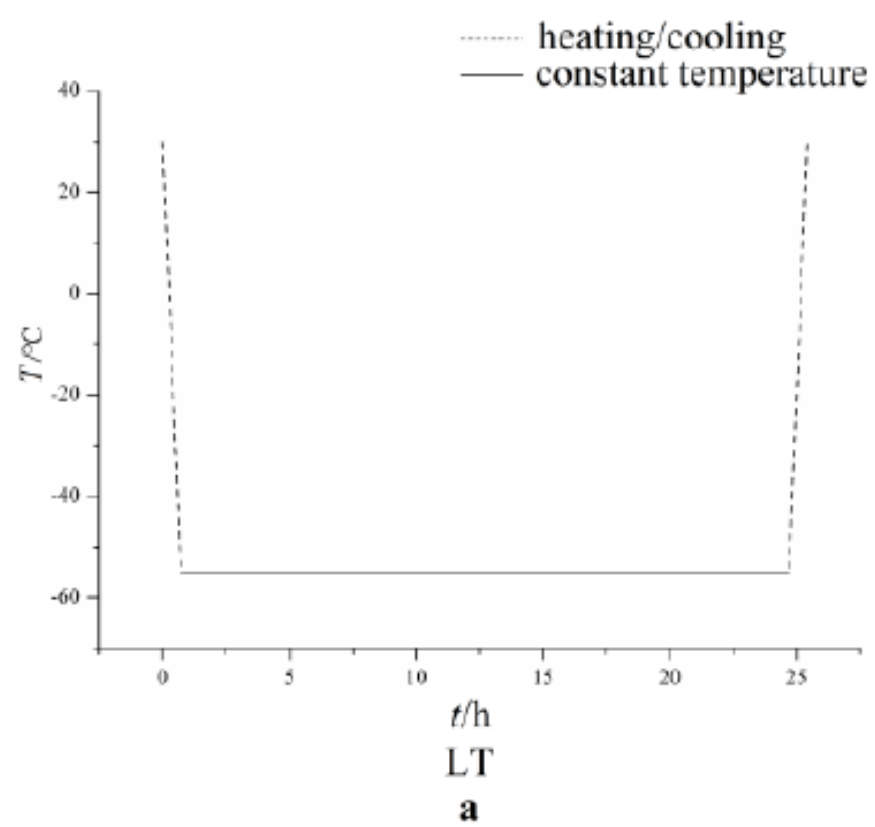

a

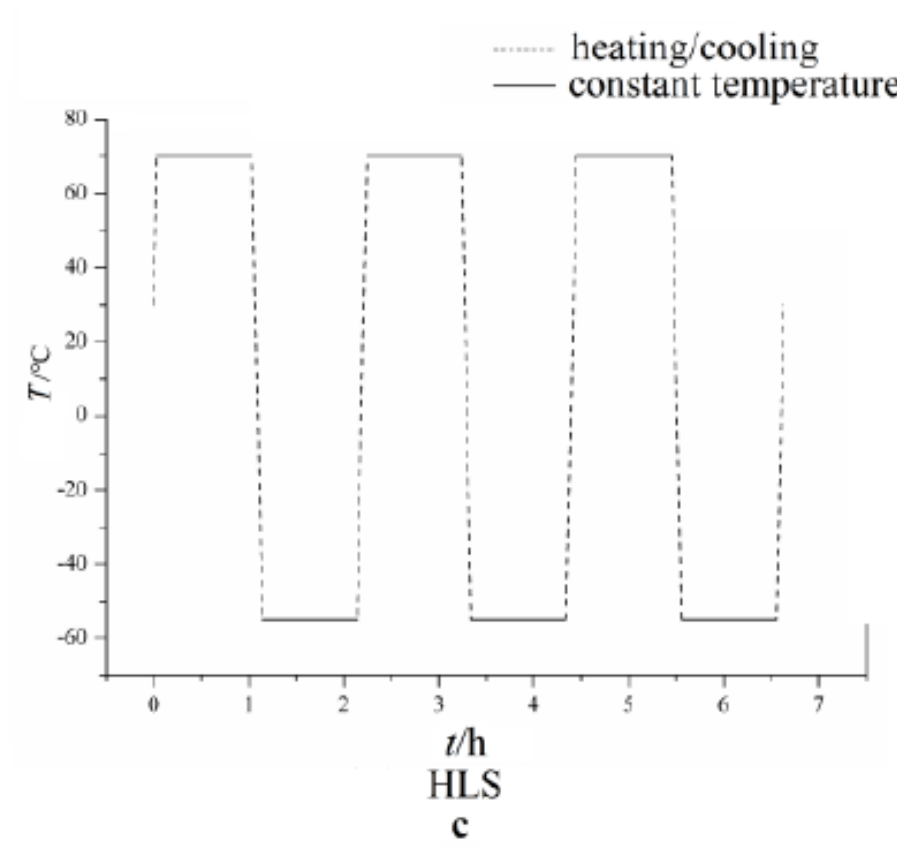

heating/cooling

constant temperature
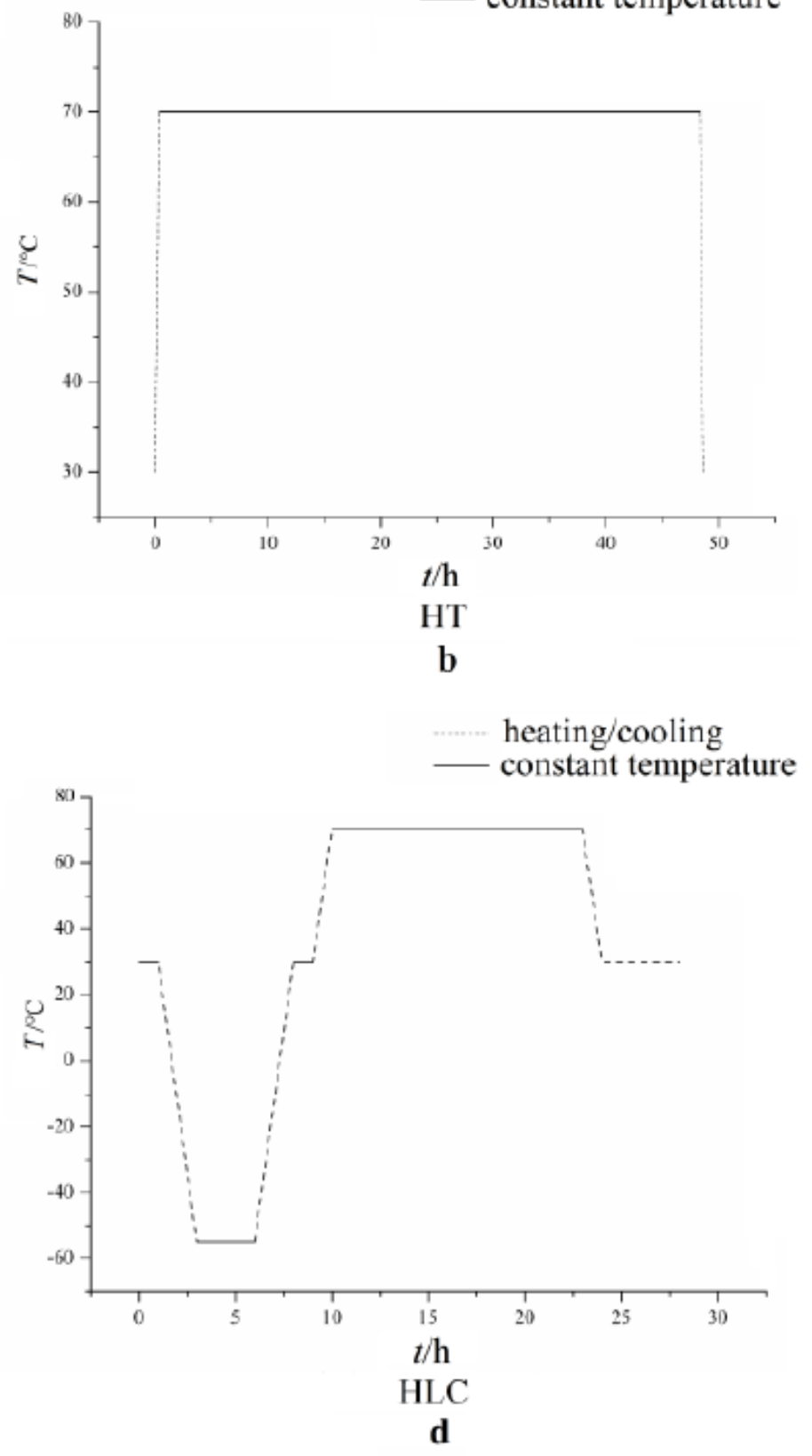

Figure 2

Temperature variation settings of different temperature adaptability treatments. 

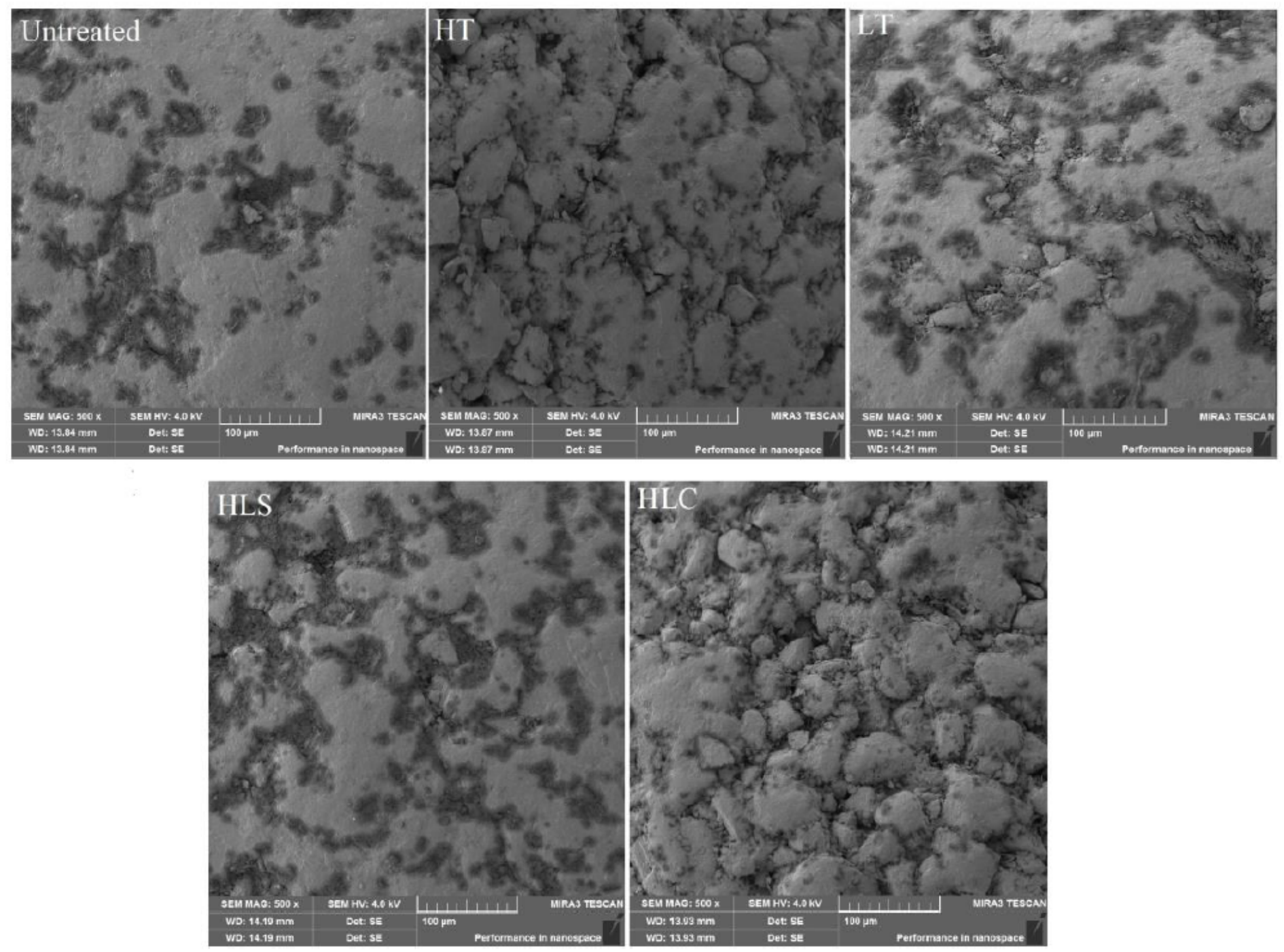

\section{Figure 3}

Surface micro-topography of the HNIW/FOX-based PBX modeling powders. 

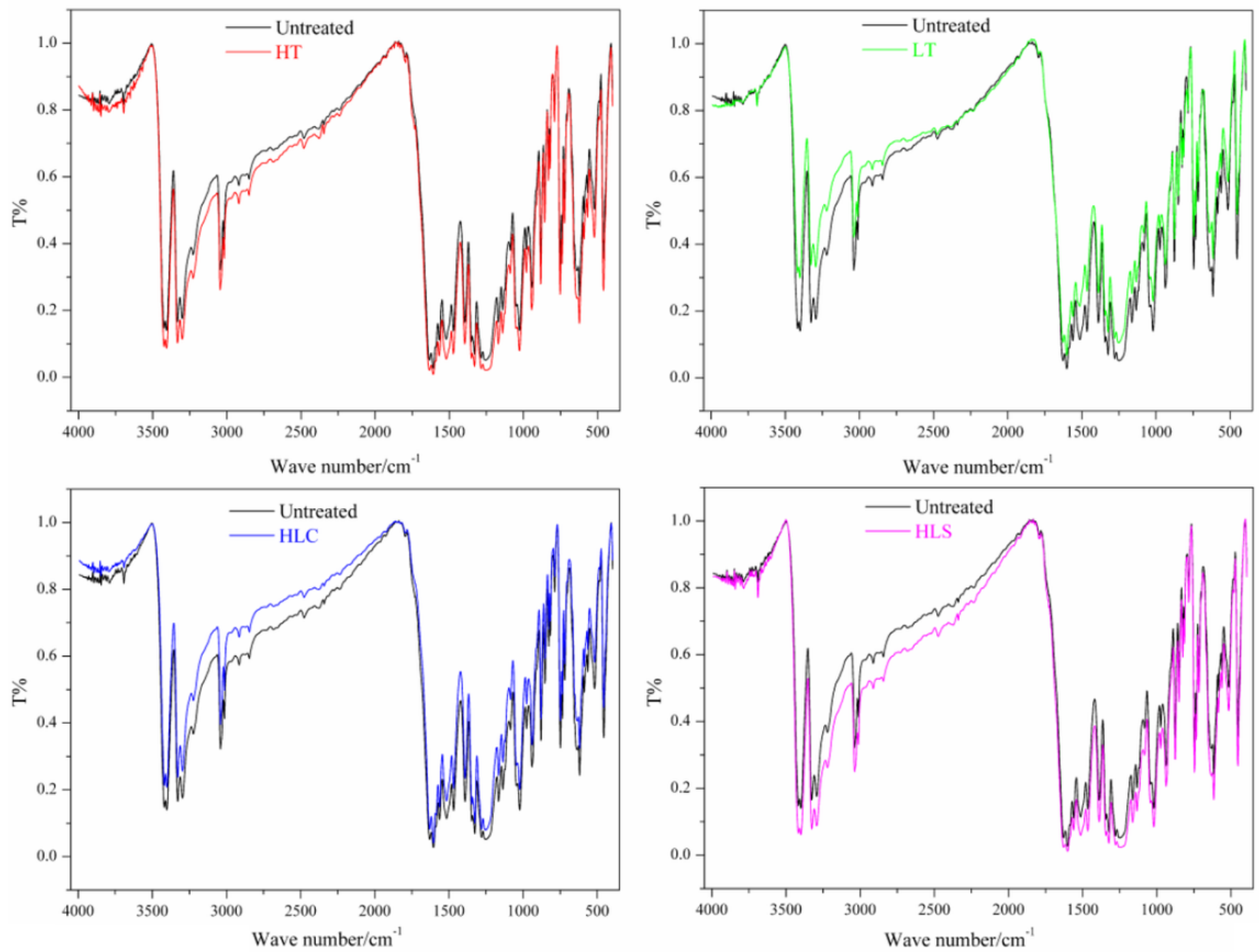

Figure 4

IR spectroscopies of HNIW/FOX-based PBX samples. 


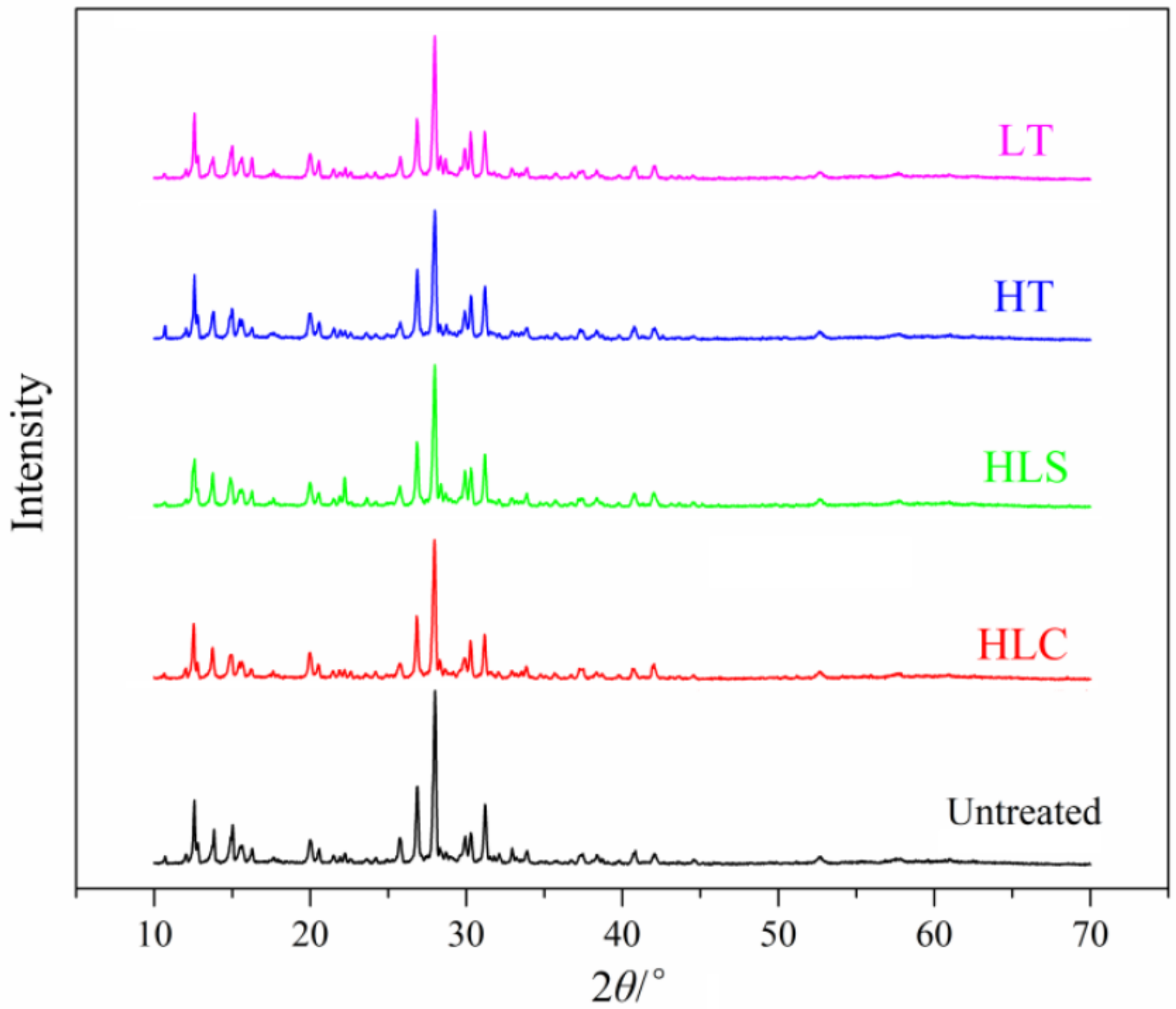

Figure 5

XRD spectroscopies of HNIW/FOX-based PBX samples. 


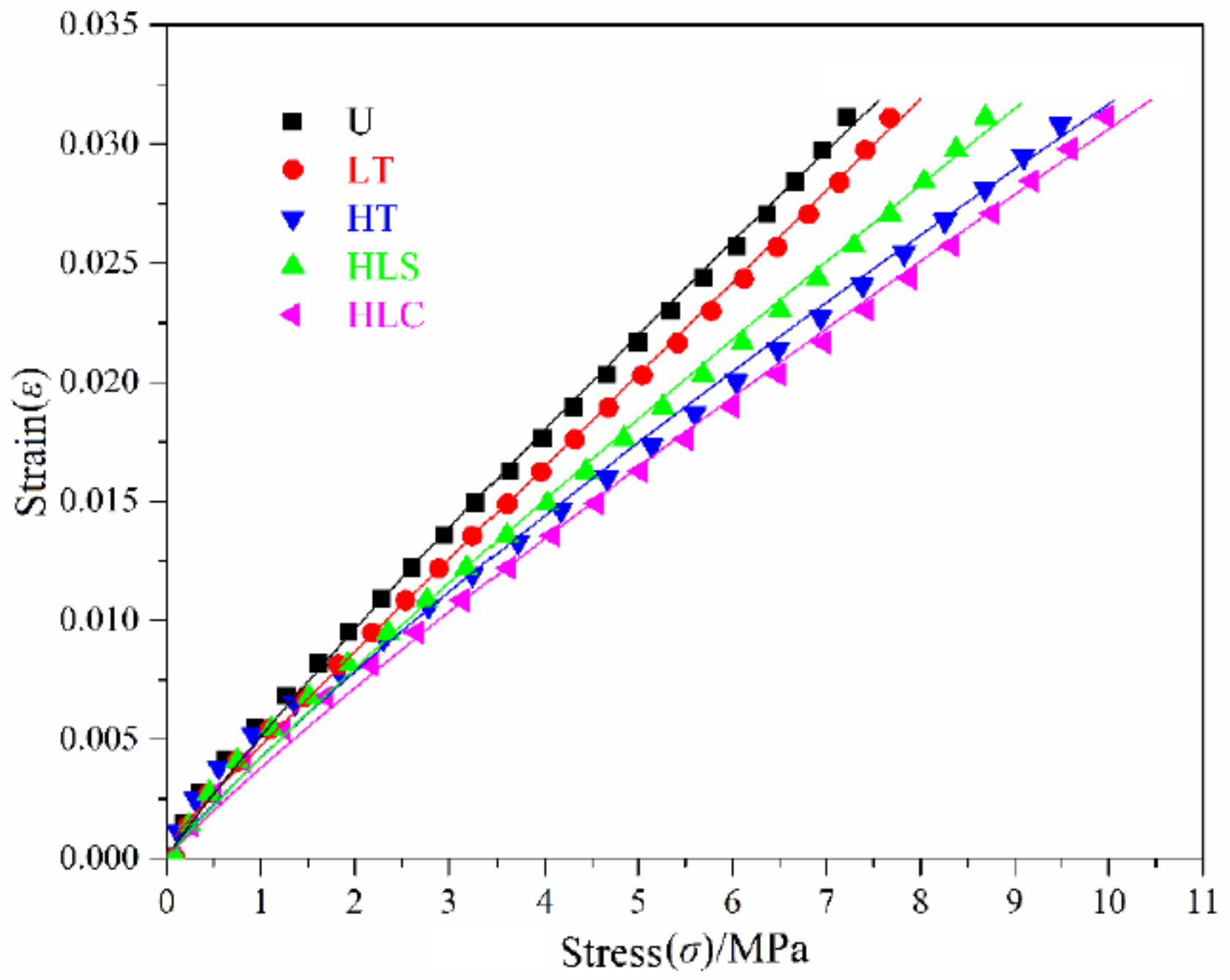

Figure 6

Stress-strain curves of HNIW/FOX-based PBX columns. 

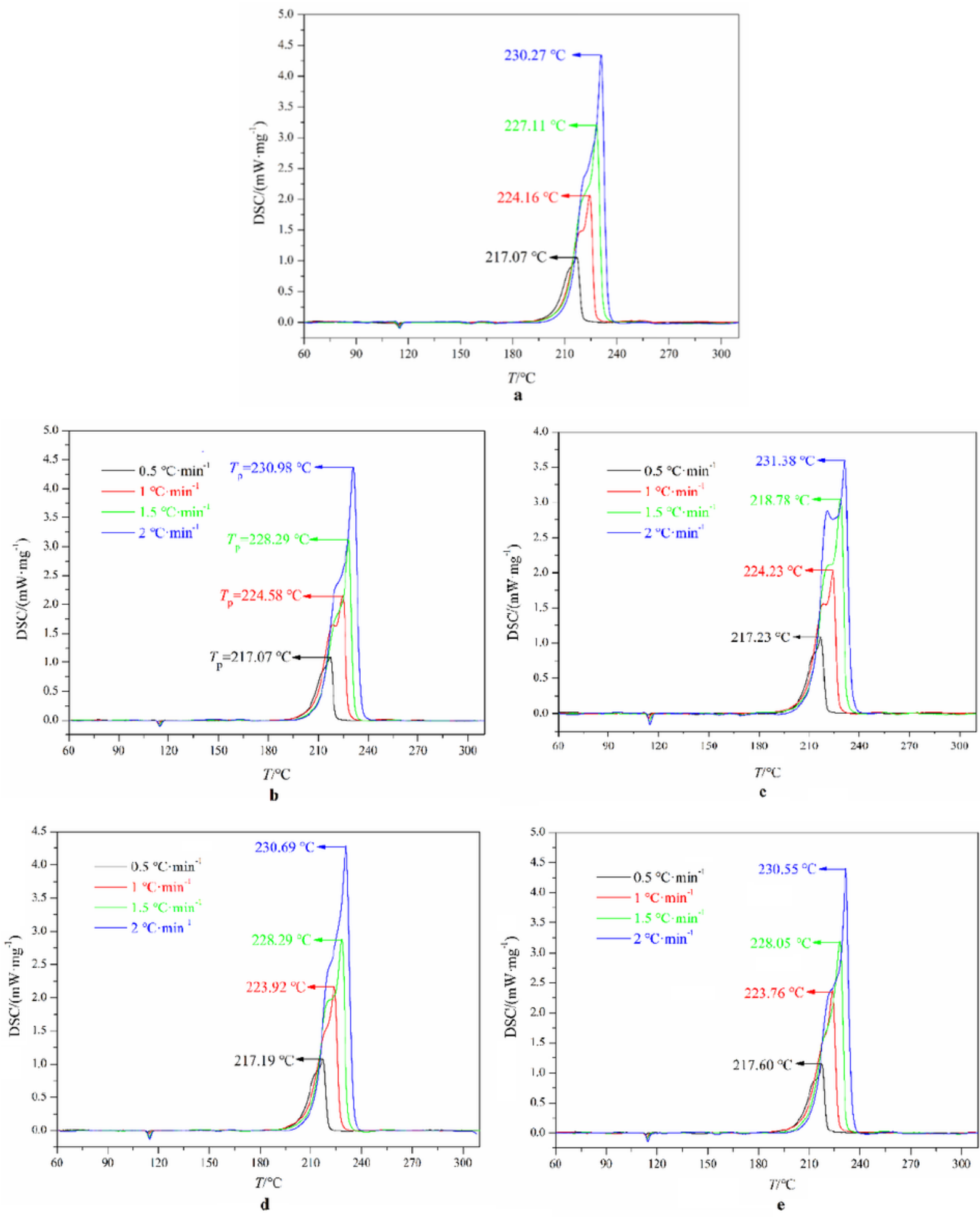

Figure 7

DSC curves of HNIW/FOX-7 based PBX samples. a untreated, b HLC treatment, c LT treatment, $d$ HT treatment, e HLS treatment 

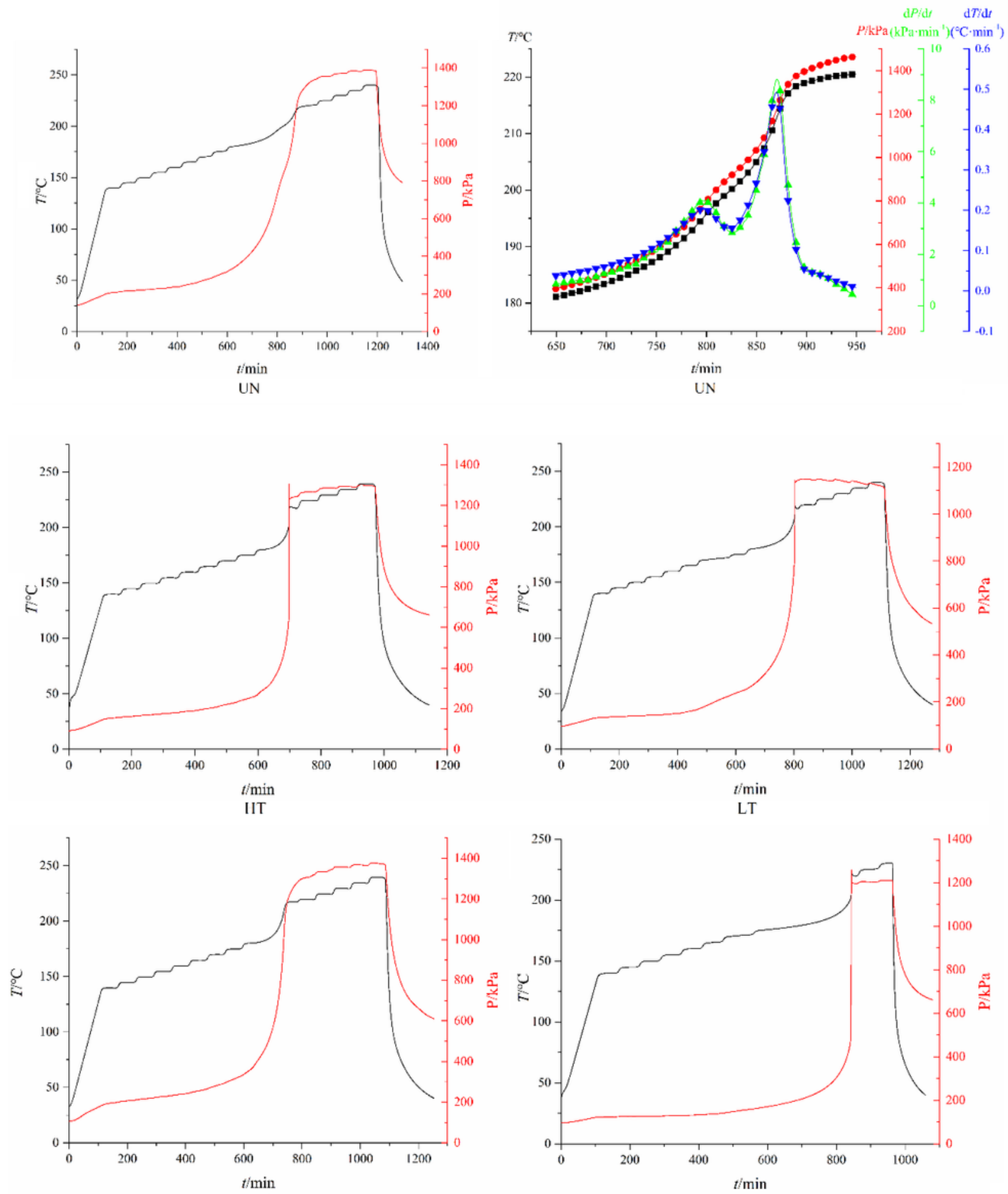

HLS

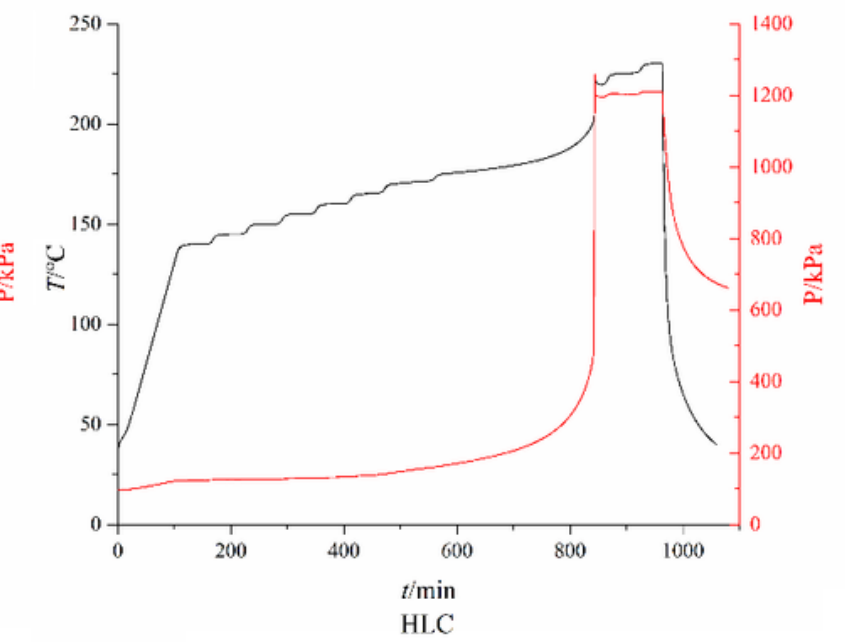

Figure 8

ARC measured results, and the variation of $\mathrm{P}, \mathrm{T}, \mathrm{dP}$ and $\mathrm{dT}$ versus $\mathrm{t}$ during the adiabatic decomposition process of untreated samples. 